\title{
A novel mechanism of neovascularization in peritoneal dissemination via cancer-associated mesothelial cells affected by TGF- $\beta$ derived from ovarian cancer
}

\author{
KAYO FUJIKAKE $^{1}$, HIROAKI KAJIYAMA ${ }^{1}$, MASATO YOSHIHARA ${ }^{1}$, KIMIHIRO NISHINO $^{1}$, \\ NOBUHISA YOSHIKAWA ${ }^{1}$, FUMI UTSUMI ${ }^{1}$, SHIRO SUZUKI ${ }^{1}$, KAORU NIIMI $^{1}$, JUN SAKATA ${ }^{1}$, \\ HIROKO MITSUI $^{2}$, KIYOSUMI SHIBATA ${ }^{3}$, TAKESHI SENGA ${ }^{4}$ and FUMITAKA KIKKAWA ${ }^{1}$
}

\author{
${ }^{1}$ Department of Obstetrics and Gynecology, Graduate School of Medicine, Nagoya University, Showa-ku, Nagoya 466-8550; \\ ${ }^{2}$ Department of Gynecology, Inuyama Chuo General Hospital, Gromaru, Inuyama; ${ }^{3}$ Department of Obstetrics \\ and Gynecology, Banbuntane Hotokukai Hospital, Fujita Health University, \\ Nagoya, Aichi; ${ }^{4}$ Yahagigawa Hospital, Anjyo 444-1164, Japan
}

Received March 29, 2017; Accepted September 8, 2017

DOI: 10.3892/or.2017.6104

\begin{abstract}
Epithelial ovarian cancer (EOC) is believed to cause peritoneum dissemination through microenvironmental cell-to-cell communication between the tumor and mesothelium, leading to the further acquisition of progressive and metastatic potentials. In the present study, we aimed to determine the role of cancer-associated mesothelial cells (CAMCs) in the promotion of tumor neovascularization and vascular permeability via enhanced vascular endothelial growth factor (VEGF) production. We examined whether a characteristic morphological change in human peritoneal mesothelial cells (HPMCs) was observed in the presence of malignant ascites and tumor-derived TGF- $\beta$. We focused on the enhanced production of VEGF in CAMCs and its crucial role in endothelial migration and tube formation. Normal HPMCs showed an epithelial morphology with a cobblestone appearance. When HPMCs were co-cultured with malignant ascites from patients with advanced EOC, a dramatic morphologic change was noted from an epithelioid pattern to an $\alpha$-SMA-positive fibroblastic, mesenchymal pattern. Additionally, we found that EOC-derived TGF- $\beta$ induced typical EMT-like morphological
\end{abstract}

Correspondence to: Dr Hiroaki Kajiyama, Department of Obstetrics and Gynecology, Graduate School of Medicine, Nagoya University, 65 Truma-cho, Showa-ku, Nagoya 466-8550, Japan E-mail: kajiyama@med.nagoya-u.ac jp

Abbreviations: CAMCs, cancer-associated mesothelial cells; EMT, epithelial-mesenchymal transition; EOC, epithelial ovarian cancer; HPMCs, human peritoneal mesothelial cells; HUVECs, human umbilical venous endothelial cells; VEGF, vascular endothelial growth factor

Key words: epithelial ovarian cancer, epithelial-mesenchymal transition, TGF- $\beta$, cancer-associated mesothelial cells, peritoneal dissemination, neovascularization alteration in HPMCs, which was associated with CAMCs. We further discovered that CAMCs play a crucial role in the enhanced migration and tube formation of endothelial cells by the promotion of VEGF production. In conclusion, our findings indicate the possible involvement of CAMCs in the neovascularization of EOC and enhancement of vascular permeability, resulting in the formation of malignant ascites. The novel mechanism of CAMCs as a facilitator of EOC progression is displayed by microenvironmental cell-to-cell communication between EOC and the mesothelium.

\section{Introduction}

Epithelial ovarian cancer (EOC) is a major cause of mortality among all gynecological malignancies (1). A recent global cancer statistics report estimated that 238,700 women were newly diagnosed with EOC and 151,900 died of this disease worldwide in 2012 (2). Peritoneal dissemination is the most common metastatic route for this tumor. At the time of diagnosis, approximately two-thirds of all EOC patients have already developed peritoneal carcinomatosis (3) and more than one-third present with malignant ascites $(4,5)$. In fact, the prognosis of patients with EOC likely depends on the degree of peritoneal dissemination (6-8). Thus, it is crucial to assess the mechanism by which detached EOC cells disseminate and implant throughout the peritoneal cavity. Indeed, an initial step in peritoneal dissemination is the attachment of tumor cells to mesothelial cells, which are the primary components of the microenvironment of EOC cells. After EOC cells attach to mesothelial cells, they invade into subperitoneal tissues with increased neovascularization (9).

Transforming growth factor- $\beta$ (TGF- $\beta$ ) is one of the important cytokines that modify the local microenvironment for tumor progression $(10,11)$. According to earlier studies, cancer-associated fibroblasts (CAFs), which are derived from normal stromal fibroblasts by the action of TGF- $\beta$, effectively promote growth, survival and progression of tumor cells $(12,13)$. Meanwhile, mesothelial cells function as a 
protective barrier against the progression of EOC cells $(14,15)$. However, similar to the production of CAFs, TGF- $\beta$ induces morphological conversion of mesothelial cells, which is associated with epithelial-mesenchymal transition (EMT) $(16,17)$, and mesothelial cells are forced to allow expansive progression of cancer cells. Indeed, in our previous study, mesothelial cells stimulated by EOC-derived TGF- $\beta$ promoted cancer cell attachment and proliferation through the downregulation of the miR-200 family and subsequent increase in fibronectin 1 (18). TGF- $\beta$-stimulated mesothelial cells have been reported to show tumor-promoting functions. In a previous study, mesothelial cells exposed to gastric cancer cells or TGF- $\beta 1$ became exfoliated and exhibited signs of injury, suggesting that they provide tumor cells with a favorable environment for peritoneal metastases (19). Considering these findings, EOC cells may spread to the peritoneum through microenvironmental cell-to-cell communication between tumor and mesothelial cells, which may contribute to the further acquisition of progressive and metastatic potentials. However, the mechanisms involved in the activation of mesothelial cells by EOC cells and the exertion of their tumor-promoting effects in the peritoneal cavity are unclear. VEGF has been implicated in the peritoneal dissemination of EOC and the subsequent development of malignant ascites (5).

In the present study, we aimed to determine the role of cancer-associated mesothelial cells (CAMCs) in the promotion of tumor neovascularization and vascular permeability via enhanced vascular endothelial growth factor (VEGF) production.

\section{Materials and methods}

Cell culture. ES-2 cells were obtained from the American Type Culture Collection (ATCC; Manassas, VA, USA) in 2012-2013. These cells were maintained in RPMI-1640 medium (Sigma, St. Louis, MO, USA) supplemented with $10 \%$ fetal bovine serum (FBS) and penicillin/streptomycin in a humidified atmosphere of $5 \% \mathrm{CO}_{2}$. Human umbilical venous endothelial cells (HUVECs) were also obtained from ATCC. These cells were maintained in EGM-2 Bullekit medium (Lonza, Walkersville, MD, USA) supplemented with $10 \% \mathrm{FBS}$ and penicillin-streptomycin at $37^{\circ} \mathrm{C}$ in a humidified atmosphere of $5 \% \mathrm{CO}_{2}$. Human peritoneal mesothelial cells (HPMCs) were isolated from surgical specimens of the human omentum. The detailed procedure for HPMC isolation has been described in our previous study (18). Briefly, small pieces of the omentum were surgically resected under sterile conditions and were trypsinized at $37^{\circ} \mathrm{C}$ for $20 \mathrm{~min}$. The suspension was then passed through a $200-\mu \mathrm{m}$ pore nylon mesh to remove undigested fragments and was centrifuged at 2,000 rpm for $5 \mathrm{~min}$. The collected cells were cultured in RPMI-1640 medium supplemented with $10 \%$ FBS. For experiments, cells were used during the second or third passage after primary culture. HPMCs were identified with immunostaining using mouse monoclonal antibodies against cytokeratin 19 and vimentin (DACO Japan Co., Ltd., Kyoto, Japan) as previously described (20). HPMCs were cultured in RPMI-1640 medium supplemented with $10 \%$ FBS and penicillin-streptomycin. The human omentum, EOC tissues, and malignant ascites of patients with EOC were obtained after receiving consent from each patient and after approval from the Ethics Committee in Nagoya University. The procedure of co-culture of HPMCs with EOC tissue has been described in detail in our previous study (17).

Western blot analysis. The western blotting procedure has been previously described (21). As primary antibodies, we used anti-E-cadherin, anti-N-cadherin, anti-vimentin, anti- $\alpha$-SMA (all from Santa Cruz Biotechnology, Santa Cruz, CA, USA), and anti- $\beta$-actin antibodies (Sigma). The primary antibodies were washed in $0.05 \%$ Tween-20/phosphate-buffered saline (PBS) and then incubated with horseradish peroxidase-conjugated secondary antibody. Proteins were visualized using an enhanced chemiluminescence reagent (Amersham Pharmacia Biotech, Piscataway, NJ, USA). Bands were visualized using ImageQuant LAS 4000 (General Electric Co., Fairfield, CT, USA).

Collection of conditioned medium (CM) from HPMCs or $C A M C s$. One hundred thousand HPMCs with or without TGF- $\beta$ treatment were seeded into 12 -well culture dishes and incubated in culture medium. After $72 \mathrm{~h}$ of incubation, the medium was replaced by fresh RPMI-1640 medium containing $1 \%$ FBS. After $24 \mathrm{~h}$ of additional incubation, the supernatant was collected and centrifuged for use in enzymelinked immunosorbent assay (ELISA).

ELISA assay for quantification of VEGF. The aforementioned culture supernatant was tested using VEGF ELISA kits (R\&D Systems, Minneapolis, MN, USA) according to the manufacturer's protocol. Experiments were performed in triplicate.

RNA extraction and quantitative real-time PCR. The procedure has been previously described (18). Total RNA was isolated using TRIzol reagent (Invitrogen, Carlsbad, CA, USA). The concentration of RNA was measured according to the absorbance at $260 \mathrm{~nm}$, and $0.5 \mu \mathrm{g}$ total RNA was reverse transcribed using M-MLV reverse transcriptase and a random primer (both from Takara, Tokyo, Japan) at $42^{\circ} \mathrm{C}$ for $60 \mathrm{~min}$. Then, $1 \mu \mathrm{l}$ of the final cDNA solution was subjected to PCR using Taq DNA polymerase (Takara). Amplification conditions were as follows: denaturation at $94^{\circ} \mathrm{C}$ for $2 \mathrm{~min} ; 28$ cycles of $94^{\circ} \mathrm{C}$ for $30 \mathrm{sec}, 50-60^{\circ} \mathrm{C}$ for $30 \mathrm{sec}$, and $72^{\circ} \mathrm{C}$ for $1 \mathrm{~min}$; and final extension at $72^{\circ} \mathrm{C}$ for $10 \mathrm{~min}$. $\beta$-actin and GAPDH were used as endogenous controls. The sequences of primers used for the experiments are as follows: VEGF forward, 5'-GGC CAGCACATAGGAGAGAT and reverse, 5'-ACGCTCCAG GACTTATACCG; fibronectin forward, 5'-GAGAATAAGCT GTACCATCGCAA and reverse, 5'-CGACCACATAGGAA GTCCCAG; Snail forward, 5'-ACTGCAACAAGGAATAC CTCAG and reverse, 5'-GCACTGGTACTTCTTGACATCTG; ZEB1 forward, 5'-GATGATGAATGCGAGTCAGATGC and reverse, 5'-CGACCACATAGGAAGTCCCAG; Slug forward, 5'-TGTGACAAGGAATATGTGAGCC and reverse, 5'-TGAG CCCTCAGATTTGACCTG; Twist forward, 5'-GTCCGCAG TCTTACGAGGAG and reverse, 5'-GCTTGAGGGTCTGAA TCTTGCT; E-cadherin forward, 5'-ATTTTTCCCTCGACAC CCGAT and reverse, 5'-TCCCAGGCGTAGACCAAGA; N-cadherin forward, 5'-TGCGGTACAGTGTAACTGGG and reverse, 5'-GAAACCGGGCTATCTGCTCG. 

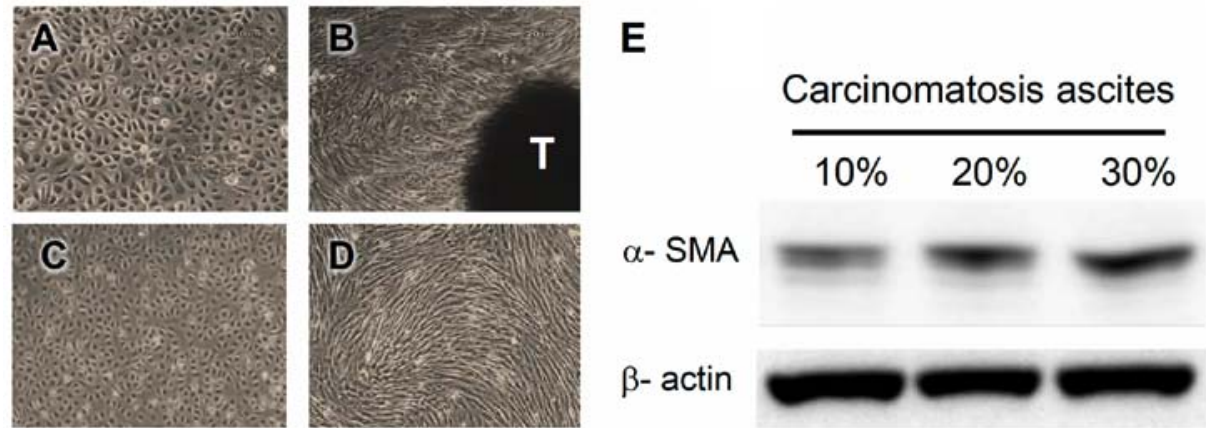

Figure 1. Morphological change in human peritoneal mesothelial cells (HPMCs) in the presence of microtissue or malignant ascites from epithelial ovarian cancer (EOC). (A) Typical morphology of cultured HPMCs: epithelial morphology with a cobblestone appearance. (B) Representative morphological conversion of HPMCs from an epithelioid, cobblestone pattern to a fibroblastic, mesenchymal pattern under co-culture with microscopic EOC tissue (T) (C and D) Representative morphology of cultured HPMCs in the (C) absence or (D) presence of malignant ascites from EOC. (E) Western blot analysis regarding the expression of $\alpha$-SMA. The $\alpha$-SMA expression was upregulated in proportion to an increase in the addition of malignant ascites. Percentages indicate the ratio of malignant ascites/total mediim volume.

In vitro migration assay. Cell migration was assayed in 24-well Transwell cell culture chambers (Costar, Corning, NY, USA). HUVECs were pretreated in EBM-2 medium (Lonza, Walkersville, MD, USA) for $1 \mathrm{~h}$. The cells were then suspended into the upper chamber at a final concentration of $5.0 \times 10^{4} / \mathrm{ml}$ in $200 \mu \mathrm{l}$ of medium. The lower chamber contained $750 \mu \mathrm{l}$ of conditioned medium from HPMCs or CAMCs (without FBS). The conditioned medium was preincubated with bevacizumab (final concentration, $150 \mu \mathrm{g} / \mathrm{ml}$ ) (Chugai Pharmaceuitcal Co., Tokyo, Japan) and/or SB-431542 (final concentration, $0.1 \mathrm{nM}$ ) (R\&D Systems, Minneapolis, $\mathrm{MN}, \mathrm{USA}$ ) for $1 \mathrm{~h}$ at $37^{\circ} \mathrm{C}$. Then, the conditioned medium was added to the lower chamber immediately before the upper chamber was inserted. After $24 \mathrm{~h}$ of incubation, the tumor cells on the upper surface of the filters were removed by wiping with cotton swabs, and the invading cells on the lower surface were stained with May-Grünwald-Giemsa stain. These cells on the lower surface of the filters were counted under a microscope at a magnification of $x 200$. We performed four individual experiments using the migration assay in triplicate.

Tube formation assay. The Matrigel tube formation assay was performed according to the instructions provided by BD Biosciences (San Jose, CA, USA). Briefly, $100 \mu 1$ of growth factor-reduced Matrigel was seeded into a 48-well plate and solidified at $37^{\circ} \mathrm{C}$ for $30 \mathrm{~min}$. HPMCs were then seeded at 30,000 cells/well on top of the Matrigel in the presence of $\mathrm{CM}$ from each group. After $6 \mathrm{~h}$ of incubation at $37^{\circ} \mathrm{C}$, tube formation was observed and counted.

TGF- $\beta$ stimulation. HPMCs were stimulated with recombinant human TGF- $\beta$ (R\&D Systems) at various concentrations in RPMI-1640 medium supplemented with 1\% FBS for each indicated time. SB-431542 (R\&D Systems) was used as an inhibitor of the TGF- $\beta$ receptor.

Statistical analysis. All data are expressed as mean \pm SD. Data were calculated from at least three independent experiments. Statistical significance of differences was analyzed using Student's t-test. A P-value of $<0.05$ was considered significant.

\section{Results}

Morphological change of HPMCs in the presence of microtissue or malignant ascites from patients with EOC. We first examined whether morphological change in HPMCs was observed in the presence of microtissue of EOC or malignant ascites from carcinomatosis patients. Normal HPMCs showed an epithelial morphology with a cobblestone appearance. In contrast, cells that were co-cultured with EOC tissue converted to a morphology with a spindle fibroblastic pattern, and such a change is typical of cells with a mesenchymal phenotype (Fig. 1A and B). Furthermore, when HPMCs were co-cultured with malignant ascites from EOC patients, the morphology changed from an epithelioid pattern to a fibroblastic, mesenchymal pattern (Fig. 1C and D). $\alpha$-SMA expression is one of the typical markers for the identification of myofibroblast (22). In western blot analysis, we noted that the expression of $\alpha$-SMA was upregulated in proportion to the increase in the added ascites (Fig. 1E, percentages indicate the ratio of malignant ascites/total medium volume).

Morphological change in HPMCs induced by CM from highly metastatic EOC cells and its blockade with a TGF- $\beta$ receptor inhibitor. In carcinomatous peritonitis of EOC, the tumor and peritoneal mesothelium are thought to influence each other. We examined whether a tumor-derived liquid factor may influence the morphology of HPMCs. ES-2 cells are a highly metastatic EOC cell line. The CM of ES-2 cells was added to cultured monolayered HPMCs. After $72 \mathrm{~h}$ of incubation, we observed marked morphological alternation in the CM-treated HPMCs from an epithelial to a mesenchymal pattern (Fig. 2A and B). This change was completely blocked by pretreatment with SB-431542, which is a TGF- $\beta$ receptor inhibitor (Fig. 2C). Thus, the effect of CM was thought to depend on at least tumor-derived TGF- $\beta$. Furthermore, the TGF- $\beta$ signaling pathway is one of the pivotal regulators of the multistep tumor microenvironment related to EMT (23-25). Thus, we further explored the expression of EMT-inducible markers, such as Snail, Fibronectin, Twist, Slug and Zeb1. We found that these EMT-related transcriptional factors were markedly upregulated by the CM-stimulated HPMCs and its effect was inhibited by SB-431542 (Fig. 2D). 

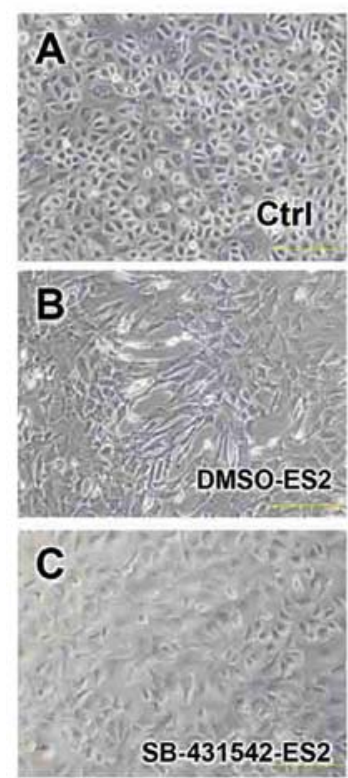

D

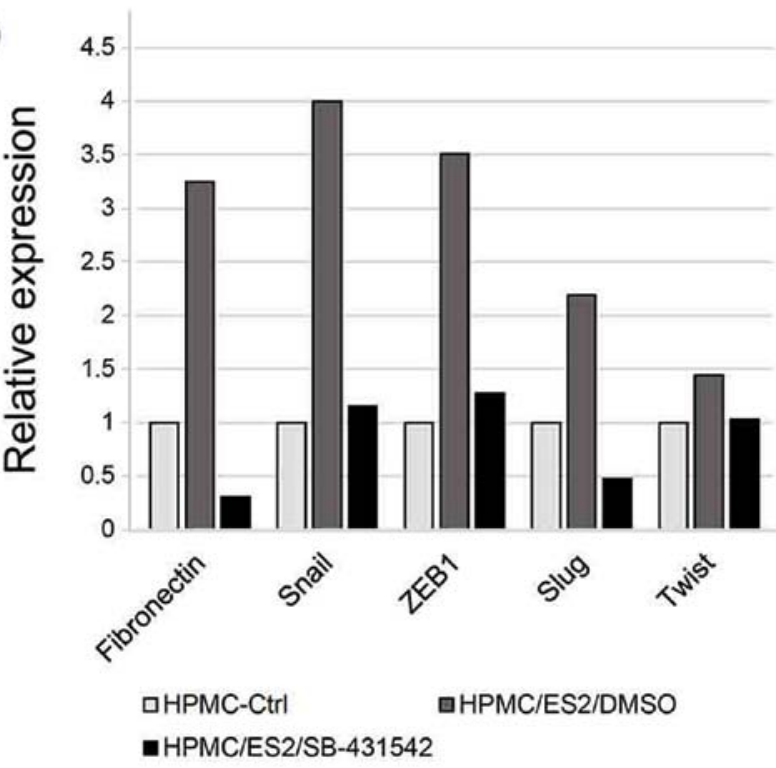

Figure 2. Morphological change in human peritoneal mesothelial cells (HPMCs) induced by conditioned medium (CM) from highly metastatic epithelial ovarian cancer cells and its blockade with a transforming growth factor (TGF)- $\beta$ receptor inhibitor. (A) Typical morphology of cultured HPMCs: epithelial morphology with a cobblestone appearance. (B) Representative morphological conversion of HPMCs from an epithelioid pattern to a mesenchymal pattern after treatment with CM from ES-2. (C) Morphology of HPMCs treated with CM in the presence of SB-431542, which is a TGF- $\beta$ receptor inhibitor (cobblestone appearance). (D) Relative mRNA expressions of epithelial-mesenchymal transition (EMT)-related transcriptional markers, such as Snail, Fibronectin, Twist, Slug and Zeb1 (quantitative real-time PCR). These EMT-related transcriptional factors were markedly upregulated by CM-stimulated HPMCs.
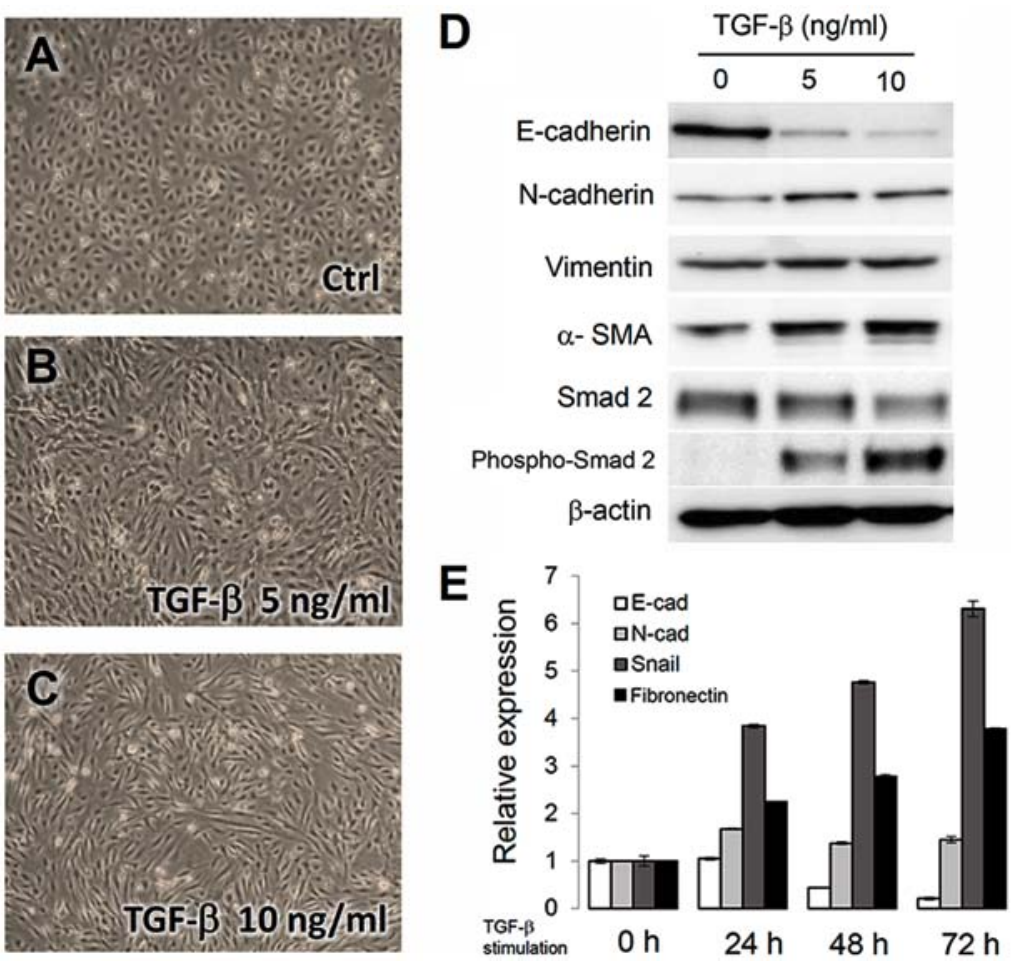

Figure 3. Transforming growth factor (TGF)- $\beta$ was responsible for a change from human peritoneal mesothelial cells (HPMCs) to cancer-associated mesothelial cells. (A) Morphological change in HPMCs in the absence or presence of TGF- $\beta$. [(A) No treatment with TGF- $\beta$; (B) $5 \mathrm{ng} / \mathrm{ml}$ of TGF- $\beta$; (C) $10 \mathrm{ng} / \mathrm{ml}$ of TGF- $\beta$; incubation time of $72 \mathrm{~h}$ ]. (D) Western blot analysis of epithelial and mesenchymal markers in HPMCs with or without stimulation with TGF- $\beta$. Upregulation of vimentin, N-cadherin and $\alpha$-SMA, and downregulation of E-cadherin were observed in TGF- $\beta$-treated HPMCs. (E) Relative mRNA expression (quantitative real-time PCR) of E-cadherin, N-cadherin, vimentin, Snail and fibronectin 1 in TGF- $\beta$-treated HPMCs at various incubation times (10 ng/ $\mathrm{ml}$ of TGF- $\beta$ ).

TGF- $\beta$ is responsible for the change from HPMCs to CAMCs. We confirmed the effects of TGF- $\beta$ on morphological features and EMT-related transcriptional factors expressed in HPMCs. The morphology of HPMCs was altered from an epithelial to 
A

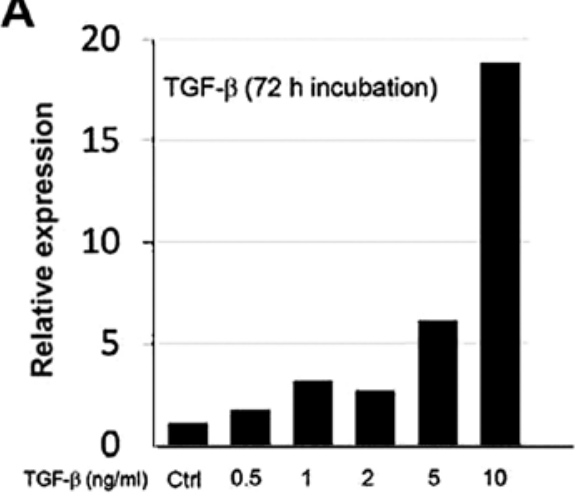

C

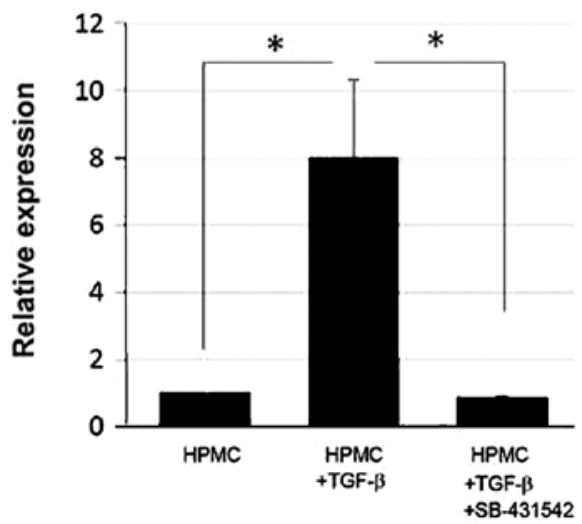

B

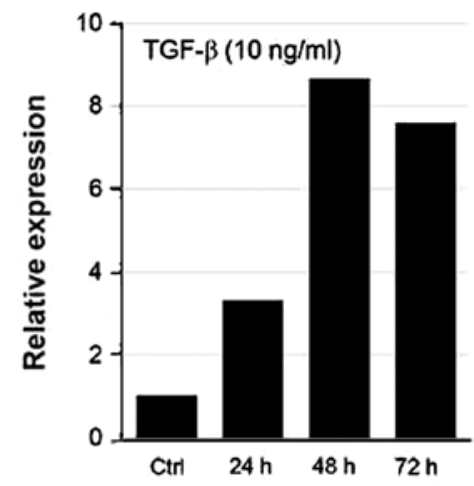

D

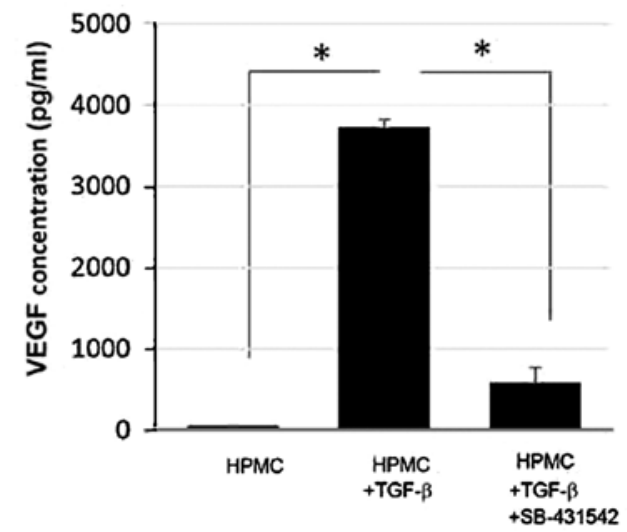

Figure 4. Upregulation of vascular endothelial growth factor (VEGF) mRNA and protein levels in cancer-associated mesothelial cells (CAMCs) and its blockade with a TGF- $\beta$ receptor inhibitor. (A) Relative mRNA levels of VEGF in the TGF- $\beta$-stimulated human peritoneal mesothelial cells (HPMCs) (CAMCs) incubated for $72 \mathrm{~h}$ at various concentrations. (B) Relative mRNA levels of VEGF in CAMCs for various incubation times. (C) Cancellation of the increased VEGF mRNA level in CAMCs by simultaneous treatment with SB-431542 (quantitative real-time PCR). Significance at *P<0.05. (D) Cancellation of the increased VEGF level in CAMCs by simultaneous treatment with SB-431542 (ELISA assay). Significance at ${ }^{*} \mathrm{P}<0.05$.

a mesenchymal pattern with TGF- $\beta$ treatment for $72 \mathrm{~h}$ (final concentration, 0,5 and $10 \mathrm{ng} / \mathrm{ml}$ in culture medium) (Fig. 3A-C). Moreover, increased expression of vimentin, $\mathrm{N}$-cadherin and $\alpha$-SMA were observed in the TGF- $\beta$-treated HPMCs, and downregulation of E-cadherin, as an epithelial marker, was identified (Fig. 3D). Similar to the results shown in Fig. 2D, the EMT-related transcriptional factors were markedly upregulated by TGF- $\beta$ stimulation (Fig. 3E). Therefore, HPMCs were forced to obtain a mesenchymal phenotype, including the expression of EMT-related molecules and characteristic morphology. The TGF- $\beta$-stimulated HPMCs were referred to as CAMCs.

Upregulation of VEGF $m R N A$ and protein levels in CAMCs and its blockade with a TGF- $\beta$ receptor inhibitor. According to a previous study, VEGF is produced by both HPMCs and EOC cells (26). We focused on VEGF expression in HPMCs and CAMCs. The expression of VEGF was upregulated by TGF- $\beta$ stimulation in a concentration-dependent manner at the mRNA level (Fig. 4A). Furthermore, stimulation with TGF- $\beta$ at $10 \mathrm{ng} /$ $\mathrm{ml}$ elevated the mRNA level of VEGF in a time-dependent manner until $48 \mathrm{~h}$ after treatment (Fig. 4B). Moreover, the increased mRNA expression of VEGF in CAMCs was abolished by simultaneous treatment with SB-431542 (Fig. 4C). The VEGF concentration in the supernatant was markedly higher for HPMCs than for CAMCs. However, the increase was abolished by treatment with SB-431542 (Fig. 4D).

Induction of higher migratory potential of HUVECs with CM from CAMCs than from HPMCs. The migration of endothelial cells is one of the characteristic features of neovascularization. Thus, we performed in vitro functional analyses with the aim of exploring the migratory potential with $\mathrm{CM}$ from CAMCs (Fig. 5A). The migratory potential of HUVECs was higher with $\mathrm{CM}$ from CAMCs than from unstimulated HPMCs (Fig. 5B-E). In addition, the migration-promoting effect was lost with the addition of bevacizumab (a VEGF inhibitor) (Fig. 5F). Furthermore, the migratory potential of HUVECs was downregulated with CM from CAMs preincubated with TGF $\beta$ R inhibitor (SB-431542) and/or bevacizumab $(\mathrm{P}<0.01)$ (Fig. 5G).

Enhanced tube formation ability of HUVECs with CM from $C A M C s$. Tube formation of endothelial cells is another characteristic feature of neovascularization. We examined the tube formation ability of HUVECs using CM from HPMCs and CAMCs in the presence or absence of bevacizumab. The tube formation capability of HUVECs was higher with CM from CAMCs than from unstimulated HPMCs. In addition, 

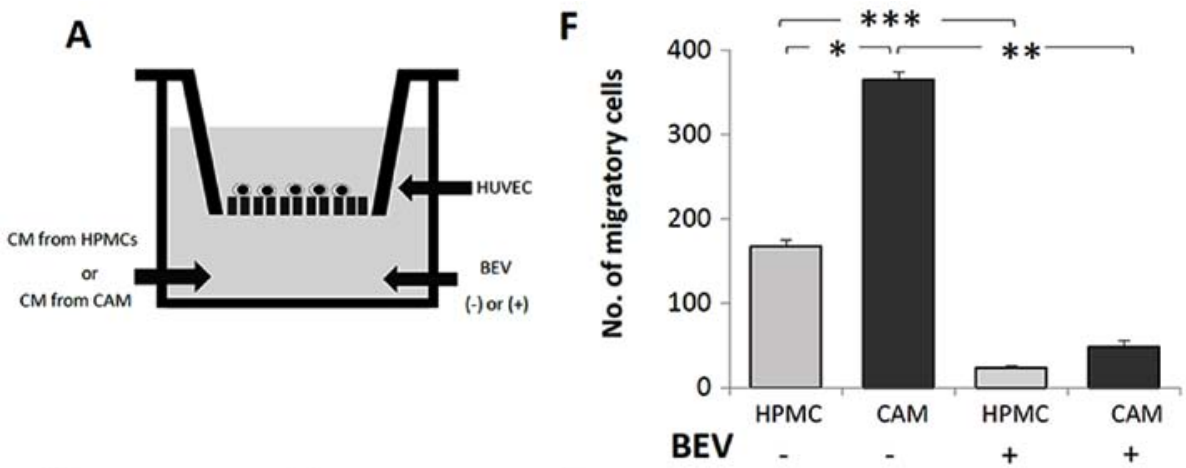

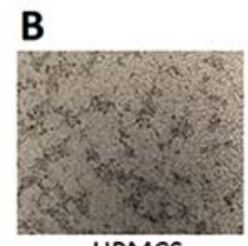

C

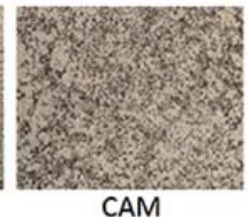

D HPMCS

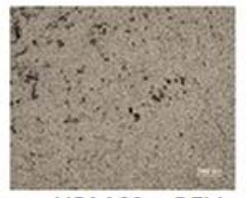

HPMCS + BEV
$\mathbf{E}$

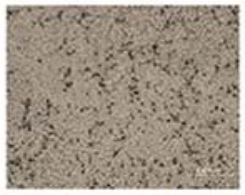

$\mathrm{CAM}+\mathrm{BEV}$
G

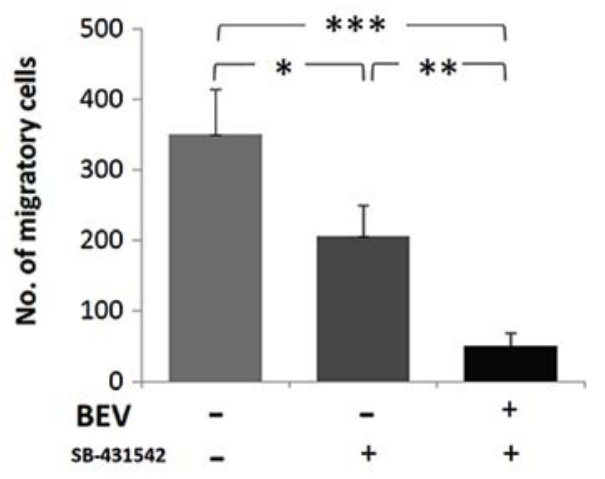

Figure 5. Induction of a higher migratory potential of human umbilical venous endothelial cells (HUVECs) with conditioned medium (CM) from cancer-associated mesothelial cells (CAMCs) than from human peritoneal mesothelial cells (HPMCs). (A) Experimental scheme using the migration assay of HUVECs (B-E) Representative images of the migrated HUVECs. Lower chamber: (B) CM from HPMCs, (C) CM from CAMCs, (D) CM from HPMCs with bevacizumab, and (E) CM from CAMCs with bevacizumab. (F) Number of migrated HUVECs. The migratory potential of HUVECs was higher with CM from CAMCs than from unstimulated HPMCs ("P<0.05). Furthermore, the migration-promoting effect was lost with bevacizumab treatment (vascular endothelial growth factor inhibitor) $\left({ }^{* * * * * *} \mathrm{P}<0.01\right)$. (G) Number of migrated HUVECs. The migratory potential of HUVECs was downregulated with CM from CAMs preincubated with TGF $\beta R$ inhibitor $(\mathrm{SB}-431542,0.1 \mathrm{nM})$ or bevacizumab $(150 \mu \mathrm{g} / \mathrm{ml})\left({ }^{*}, * * * * * * * \mathrm{P}<0.01\right)$.

A

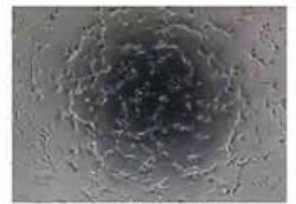

CM of HPMCS

C

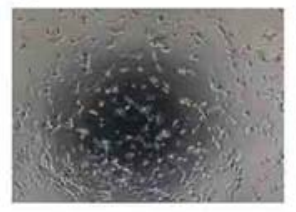

$\mathrm{CM}$ of HPMCS + BEV
B

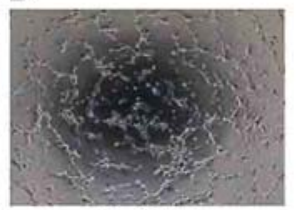

CM of CAM

D

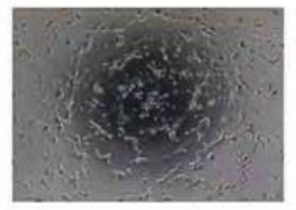

$\mathrm{CM}$ of $\mathrm{CAM}+\mathrm{BEV}$
E

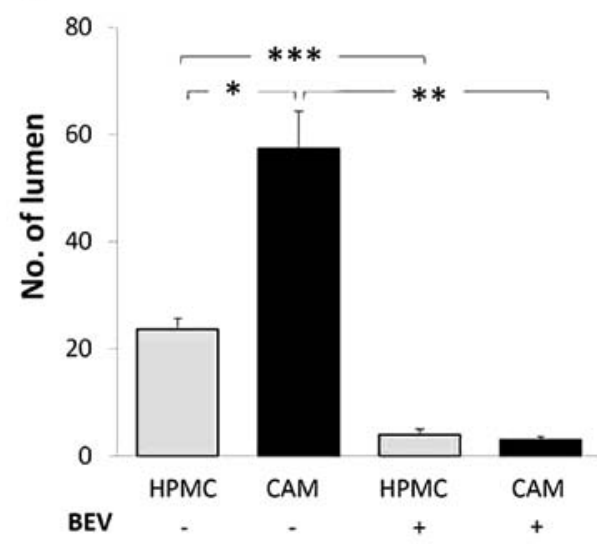

Figure 6. Enhanced tube formation ability of human umbilical venous endothelial cells (HUVECs) with conditioned medium (CM) from cancer-associated mesothelial cells (CAMCs). (A-D) Representative images of tube formation in HUVECs with CM from human peritoneal mesothelial cells (HPMCs) or CAMCs in the presence or absence of bevacizumab. (A) CM from HPMCs. (B) CM from CAMCs. (C) CM from HPMCs with bevacizumab. (D) CM from CAMCs with bevacizumab. (E) Number of tube forming HUVECs. The tube formation ability of HUVECs was higher with CM from CAMCs than from HPMCs $\left({ }^{*} \mathrm{P}<0.05\right)$. Furthermore, the tube-formation effect was lost with bevacizumab treatment (vascular endothelial growth factor inhibitor) $\left({ }^{* * * * * *} \mathrm{P}<0.01\right)$.

this effect was almost completely blocked by treatment with bevacizumab (Fig. 6A-E).

\section{Discussion}

Peritoneal metastasis is the most frequent clinical presentation of EOC, and it includes the following multiple steps: release of tumor cells from the original ovarian neoplasm, attachment to the mesothelium and subsequent migration/invasion into the subperitoneal tissue. HPMCs are an important counterpart of EOC with regard to peritoneal metastasis. However, HPMCs in themselves are not constitutionally part of tumor cells. The morphology of HPMCs represents epithelial features and so-called cobblestone appearance with tight cell-cell adhesion 
as a protective barrier of the peritoneal surface. HPMCs play a pivotal role in the maintenance and preservation of the seromucous peritoneum and production of a variety of bioactive substances, including inflammatory cytokines, growth factors and extracellular matrix components. However, phenotypic and EMT-associated morphological changes have been observed in response to several biological stimulations $(16,27)$.

In the present study, we showed that the morphology of HPMCs was markedly altered from an epithelioid pattern to a fibroblastic, mesenchymal pattern with increased expression of $\alpha$-SMA when the cells were co-cultured with malignant ascites from EOC patients. In addition, a similar morphological alteration was observed in the presence of the supernatant of highly metastatic EOC cells. Nevertheless, the effect was completely reversed by pretreatment with a TGF- $\beta$ receptor inhibitor, suggesting that tumor-derived TGF- $\beta$ was associated with the morphology of HPMCs. Thus, our findings support the notion that tumor-derived TGF- $\beta$ functions as a pivotal mediator of the interaction between cancer cells and HPMCs. Actually, TGF- $\beta$ induced phenotypic changes, including acquisition of mesenchymal morphology and EMT-like characteristics, which were associated with upregulation of EMT-inducible factors. However, the morphological change in HPMCs was previously observed and recognized as 'mesothelial-mesenchymal transition' $(16,27)$. TGF- $\beta$ has been reported to be a multifunctional cytokine, which regulates a number of cellular processes, such as proliferation, apoptosis induction, differentiation and motility (28). Additionally, TGF- $\beta$ was clinically overexpressed in EOC tissues, and an elevated TGF- $\beta$ level was identified in malignant ascites associated with this disease (29). These findings prompted us to hypothesize that the interaction between EOC cells and HPMCs is similar to that between a tumor and CAFs. Thus, in the present study, we called TGF- $\beta$-stimulated HPMCs as CAMCs.

VEGF is a key modulator of the development of intraperitoneal dissemination associated with EOC through acceleration of both neovascularization and ascites-causing vascular permeability, resulting in the further development of peritoneal carcinomatosis (5). In an earlier experimental model of EOC, inhibition of TGF- $\beta$ in tumor cells helped reduce the production of ascites through blockade of VEGF production (30). In the present study, CAMCs exerted an unregulated migratory potential and tube-forming capability on HUVECs through an increase in the production of VEGF. These results support the hypothesis that the angiogenesis/permeability effects were promoted by CAMCs in accordance with tumor spread. More specifically, the subperitoneal space is primarily composed of fatty tissue with associated nerves, vessels and lymphatic tissues having serosal layers, which make up the ligaments and mesenteries associated with the major abdominal organs and viscera (31). Additionally, the subperitoneal space is abundant in numerous capillary vessels and lymph capillaries. VEGF produced by CAMCs may act in endothelial cells located in subperitoneal tissues and promote their migration and development into microvessels to facilitate tumor angiogenesis. Clinically, treatment with bevacizumab, which is an antagonist of VEGF, suppresses the formation of malignant ascites, leading to patient relief (32). The mechanism by which bevacizumab negatively controls the accumulation of ascites remains

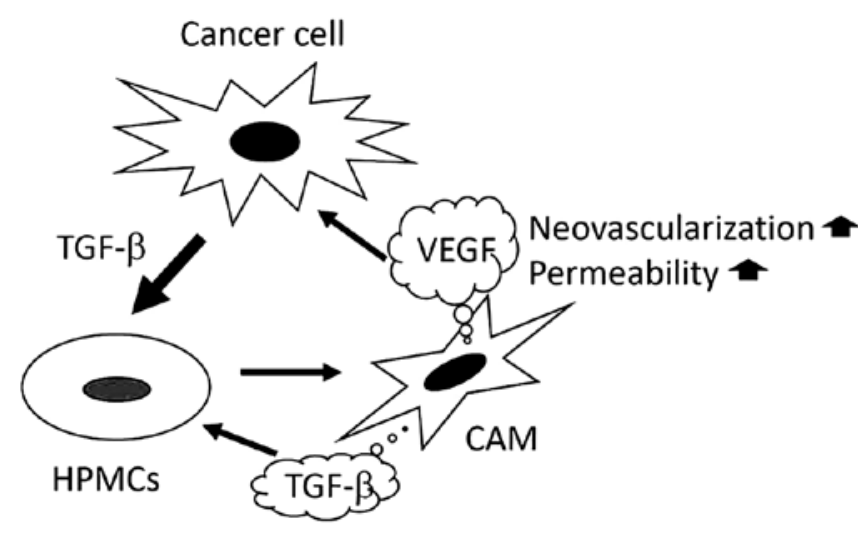

Figure 7. Scheme of the triangle axis among cancer, ascites and the mesothelium.

unclear. Probably, bevacizumab may suppress CAMC-derived VEGF as well as tumor-derived VEGF, resulting in a marked reduction of peritoneal permeability.

To date, it has been mainly thought that EOCs themselves trigger VEGF production along with VEGFR-2 expression, which works in an autocrine fashion to enhance tumor growth (33). However, according to several studies HPMCs likewise synthesize VEGF as a second source in response to stimulation by several cytokines, which is consistent with our data $(26,34)$. These observations led us to consider that VEGF production in HPMCs is crucial for the development of carcinomatosis in EOC. The mesothelium widely covers the surface of the intraperitoneal cavity. Accordingly, tumor-derived bioactive substances, such as TGF- $\beta$, broadly spread over all areas of the mesothelium via ascites and may gradually induce the change from HPMCs to CAMCs. In contrast, in our earlier study, an increase in exogenous TGF- $\beta$ induced the additional production of endogenous TGF- $\beta$ from HPMCs, resulting in the synergistic accumulation of TGF- $\beta$ (16). Thus, CAMCs subsequently secrete TGF- $\beta$ and VEGF in turn, leading to the further production of malignant ascites. In the present study, the migratory-promoting effect of endothelial cells by CAM was downregulated in the presence of a TGF $\beta$ R inhibitor as well as VEGF inhibitor. The possible explanation for such a phenomenon was based on the existence of endogenous TGF- $\beta$ from CAMs and its enhanced effect of endothelial migration. These findings indicate the scheme of the auto-stimulatory triangle axis among cancer, ascites and the mesothelium (Fig. 7). This novel hypothesis may be one of the synergic mechanisms of the development of carcinomatosis peritonitis in EOC. We believe that our findings contribute to the better understanding of the pathogenesis of EOC progression in the peritoneal cavity.

In conclusion, we proposed a possible scheme of TGF- $\beta$ associated molecular cell-to-cell communication between EOC cells and CAMCs in the microenvironmental neovascularization of the peritoneal surface. Tumor-derived TGF- $\beta$ stimulates the phenotypic change of mesothelial cells from normal HPMCs to CAMCs, along with the upregulation of VEGF production, which may facilitate tumor progression via enhanced angiogenesis and production of malignant ascites. We should recognize that the peritoneal mesothelium could be 
the largest origin of VEGF production in the pathogenesis of EOC carcinomatosis as it widely occupies the entire peritoneal surface. Comprehension of the molecular cross-talk between the tumor and the mesothelium in EOC may help in the development of a novel strategic treatment for EOC and lead to survival prolongation among patients with EOC.

\section{References}

1. Brun JL, Feyler A, Chêne G, Saurel J, Brun G and Hocké C: Long-term results and prognostic factors in patients with epithelial ovarian cancer. Gynecol Oncol 78: 21-27, 2000.

2. Torre LA, Bray F, Siegel RL, Ferlay J, Lortet-Tieulent J and Jemal A: Global cancer statistics, 2012. CA Cancer J Clin 65: 87-108, 2015.

3. Muñoz-Casares FC, Rufián S, Arjona-Sánchez Á, Rubio MJ, Díaz R, Casado Á, Naranjo Á, Díaz-Iglesias CJ, Ortega R, Muñoz-Villanueva MC, et al: Neoadjuvant intraperitoneal chemotherapy with paclitaxel for the radical surgical treatment of peritoneal carcinomatosis in ovarian cancer: A prospective pilot study. Cancer Chemother Pharmacol 68: 267-274, 2011.

4. Meunier L, Puiffe ML, Le Page C, Filali-Mouhim A, Chevrette M, Tonin PN, Provencher DM and Mes-Masson AM Effect of ovarian cancer ascites on cell migration and gene expression in an epithelial ovarian cancer in vitro model. Transl Oncol 3: 230-238, 2010

5. Masoumi Moghaddam S, Amini A, Morris DL and Pourgholami MH: Significance of vascular endothelial growth factor in growth and peritoneal dissemination of ovarian cancer. Cancer Metastasis Rev 31: 143-162, 2012.

6. Thiery JP: Epithelial-mesenchymal transitions in tumour progression. Nat Rev Cancer 2: 442-454, 2002.

7. Boyer B, Vallés AM and Edme N: Induction and regulation of epithelial-mesenchymal transitions. Biochem Pharmacol 60: 1091-1099, 2000.

8. Vernon AE and LaBonne C: Tumor metastasis: A new twist on epithelial-mesenchymal transitions. Curr Biol 14: R719-R721, 2004.

9. Terauchi M, Kajiyama H, Yamashita M, Kato M, Tsukamoto H, Umezu T, Hosono S, Yamamoto E, Shibata K, Ino K, et al: Possible involvement of TWIST in enhanced peritoneal metastasis of epithelial ovarian carcinoma. Clin Exp Metastasis 24: 329-339, 2007.

10. Drabsch Y and ten Dijke P: TGF- $\beta$ signalling and its role in cancer progression and metastasis. Cancer Metastasis Rev 31: 553-568, 2012.

11. Pickup M, Novitskiy S and Moses HL: The roles of TGF $\beta$ in the tumour microenvironment. Nat Rev Cancer 13: 788-799, 2013.

12. Yu Y, Xiao CH, Tan LD, Wang QS, Li XQ and Feng YM: Cancerassociated fibroblasts induce epithelial-mesenchymal transition of breast cancer cells through paracrine TGF- $\beta$ signalling. Br J Cancer 110: 724-732, 2014.

13. Giannoni E, Bianchini F, Masieri L, Serni S, Torre E, Calorini L and Chiarugi P: Reciprocal activation of prostate cancer cells and cancer-associated fibroblasts stimulates epithelial-mesenchymal transition and cancer stemness. Cancer Res 70: 6945-6956, 2010

14. Kenny HA, Krausz T, Yamada SD and Lengyel E: Use of a novel 3D culture model to elucidate the role of mesothelial cells, fibroblasts and extra-cellular matrices on adhesion and invasion of ovarian cancer cells to the omentum. Int J Cancer 121: 1463-1472, 2007.

15. Kenny HA, Dogan S, Zillhardt M, K Mitra A, Yamada SD, Krausz T and Lengyel E: Organotypic models of metastasis: A three-dimensional culture mimicking the human peritoneum and omentum for the study of the early steps of ovarian cancer metastasis. Cancer Treat Res 149: 335-351, 2009.

16. Kajiyama H, Shibata K, Ino K, Nawa A, Mizutani S and Kikkawa F: Possible involvement of SDF-1alpha/CXCR4-DPPIV axis in TGF-beta1-induced enhancement of migratory potential in human peritoneal mesothelial cells. Cell Tissue Res 330: 221-229, 2007.

17. Kajiyama H, Shibata K, Terauchi M, Ino K, Nawa A and Kikkawa F: Involvement of SDF-1alpha/CXCR4 axis in the enhanced peritoneal metastasis of epithelial ovarian carcinoma Int J Cancer 122: 91-99, 2008.
18. Sugiyama K, Kajiyama H, Shibata K, Yuan H, Kikkawa F and Senga T: Expression of the miR200 family of microRNAs in mesothelial cells suppresses the dissemination of ovarian cancer cells. Mol Cancer Ther 13: 2081-2091, 2014.

19. Na D, Lv ZD, Liu FN, Xu Y, Jiang CG, Sun Z, Miao ZF, Li F and $\mathrm{Xu}$ HM: Transforming growth factor beta1 produced in autocrine/paracrine manner affects the morphology and function of mesothelial cells and promotes peritoneal carcinomatosis. Int J Mol Med 26: 325-332, 2010.

20. Kajiyama H, Kikkawa F, Maeda O, Suzuki T, Ino K and Mizutani S: Increased expression of dipeptidyl peptidase IV in human mesothelial cells by malignant ascites from ovarian carcinoma patients. Oncology 63: 158-165, 2002.

21. Hosono S, Kajiyama H, Terauchi M, Shibata K, Ino K, Nawa A and Kikkawa F: Expression of Twist increases the risk for recurrence and for poor survival in epithelial ovarian carcinoma patients. Br J Cancer 96: 314-320, 2007.

22. Desmoulière $A$, Guyot $C$ and Gabbiani G: The stroma reaction myofibroblast: A key player in the control of tumor cell behavior. Int J Dev Biol 48: 509-517, 2004.

23. Crosas-Molist E, Bertran E and Fabregat I: Cross-talk between TGF- $\beta$ and NADPH oxidases during liver fibrosis and hepatocarcinogenesis. Curr Pharm Des 21: 5964-5976, 2015.

24. Dhanasekaran R, Nakamura I, Hu C, Chen G, Oseini AM, Seven ES, Miamen AG, Moser CD, Zhou W, van Kuppevelt TH, et al: Activation of the transforming growth factor- $\beta / \mathrm{SMAD}$ transcriptional pathway underlies a novel tumor-promoting role of sulfatase 1 in hepatocellular carcinoma. Hepatology 61 : 1269-1283, 2015

25. Liu J, Chen S, Wang W, Ning BF, Chen F, Shen W, Ding J, Chen W, Xie WF and Zhang X: Cancer-associated fibroblasts promote hepatocellular carcinoma metastasis through chemokine-activated hedgehog and TGF- $\beta$ pathways. Cancer Lett 379: 49-59, 2016.

26. Sako A, Kitayama J, Yamaguchi H, Kaisaki S, Suzuki H, Fukatsu K, Fujii S and Nagawa H: Vascular endothelial growth factor synthesis by human omental mesothelial cells is augmented by fibroblast growth factor-2: Possible role of mesothelial cell on the development of peritoneal metastasis. J Surg Res 115: 113-120, 2003.

27. Yáñez-Mó M, Lara-Pezzi E, Selgas R, Ramírez-Huesca M, Domínguez-Jiménez C, Jiménez-Heffernan JA, Aguilera A, Sánchez-Tomero JA, Bajo MA, Alvarez V, et al: Peritoneal dialysis and epithelial-to-mesenchymal transition of mesothelial cells. N Engl J Med 348: 403-413, 2003.

28. Massagué J, Blain SW and Lo RS: TGFbeta signaling in growth control, cancer, and heritable disorders. Cell 103: 295-309, 2000.

29. Santin AD, Bellone S, Ravaggi A, Roman J, Smith CV, Pecorelli S, Cannon MJ and Parham GP: Increased levels of interleukin-10 and transforming growth factor-beta in the plasma and ascitic fluid of patients with advanced ovarian cancer. BJOG 108: 804-808, 2001.

30. Liao S, Liu J, Lin P, Shi T, Jain RK and Xu L: TGF-beta blockade controls ascites by preventing abnormalization of lymphatic vessels in orthotopic human ovarian carcinoma models. Clin Cancer Res 17: 1415-1424, 2011.

31. Silverman PM: The subperitoneal space: Mechanisms of tumour spread in the peritoneal cavity, mesentery, and omentum. Cancer Imaging 4: 25-29, 2003.

32. Pujade-Lauraine E, Hilpert F, Weber B, Reuss A, Poveda A, Kristensen G, Sorio R, Vergote I, Witteveen P, Bamias A, et al: Bevacizumab combined with chemotherapy for platinum-resistant recurrent ovarian cancer: The AURELIA open-label randomized phase III trial. J Clin Oncol 32: 1302-1308, 2014.

33. Boocock CA, Charnock-Jones DS, Sharkey AM, McLaren J, Barker PJ, Wright KA, Twentyman PR and Smith SK: Expression of vascular endothelial growth factor and its receptors flt and KDR in ovarian carcinoma. J Natl Cancer Inst 87: 506-516, 1995.

34. Stadlmann S, Amberger A, Pollheimer J, Gastl G, Offner FA, Margreiter R and Zeimet AG: Ovarian carcinoma cells and IL-1beta-activated human peritoneal mesothelial cells are possible sources of vascular endothelial growth factor in inflammatory and malignant peritoneal effusions. Gynecol Oncol 97: 784-789, 2005. 